\title{
Estimativa da radiação solar global em função da temperatura do ar e isolinhas para o Estado de Mato Grosso do Sul, Brasil
}

\section{Soetânia Santos de Oliveira ${ }^{1}$, Guilherme Henrique Cavazzana $^{2}$ e Amaury de Souza ${ }^{2}$}

1Faculdades Integradas de Patos. Rua Horácio Nóbrega, S/№. Patos-PB, Brasil (CEP 58704-000). E-mail: soetania@gmail.com.

${ }^{2}$ Universidade Federal de Mato Grosso do Sul. Av. Fernando Correa da Costa, S/№. Cuiabá-MT, Brasil (CEP 78060-900).

Resumo. A radiação solar incidente $\left(Q_{\mathrm{g}}\right)$ é uma variável importante em estudos agrícolas, particularmente para a estimativa da evapotranspiração e em modelos para produtividade, assim como para produção de energia. Entretanto, sua medição não é, em geral, realizada em estações meteorológicas convencionais. 0 objetivo deste trabalho foi avaliar dois modelos empíricos de estimativa de $\left(\mathrm{Q}_{\mathrm{g}}\right)$, a partir da temperatura, e adicionalmente traçar as isolinhas para o Estado de Mato Grosso do Sul. Dados de $Q_{\mathrm{g}}$ diários foram obtidos por estações meteorológicas automáticas instaladas ao longo do estado e pertencentes ao Instituto Nacional de Meteorologia (INMET). Para os dois modelos foram gerados coeficientes locais de calibração. 0 desempenho de cada método foi avaliado através dos seguintes indicadores: o Viés, o erro quadrático médio (EQM), o erro absoluto médio (EA) e o índice de correlação $(r)$. Os resultados mostraram que o Modelo 1 teve um melhor desempenho, apresentando um coeficiente de correlação de 0,77 e EQM e EA inferiores a 1,8 MJ. Isto significa que tal modelo é passível de utilização para estimativa de radiação solar, mas, dado à simplicidade, desempenho e significância.

Palavras-chave: Radiação solar; Dados climáticos; Temperatura do ar; Estimativa da radiação solar.

Abstract. Estimation of global solar radiation as a function of
air temperature and isolines for the State of Mato Grosso do
Sul, Brazil. Incident solar radiation (Qg) is an important variable
in agricultural studies, particularly for the estimation of
evapotranspiration and models for productivity, as well as for
energy production. However, its measurement is not usually
performed in conventional meteorological stations. The objective
of this work was to evaluate two empirical models of (Qg)
estimation, from the temperature, and additionally to draw the
isolines to the State of Mato Grosso do Sul. Daily (Qg) data were
obtained by automatic meteorological stations installed in these

Recebido:

20/01/2019

Aceito:

19/03/2019

Publicado:

$30 / 04 / 2019$

Acesso aberto

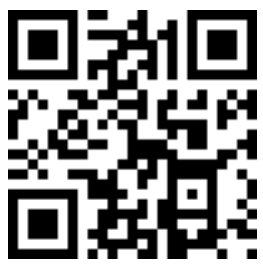

ORCID

(1) 0000-0001-8885-0998

Soetânia Santos de Oliveira

(1) 0000-0001-6878-3687

Guilherme Henrique

Cavazzana

(1) 0000-0001-8168-1482

Amaury de Souza 
regions and belonging to the National Institute of Meteorology (INMET). For both models, local calibration coefficients were generated. The performance of each method was evaluated through the following indicators: bias, root mean square error (RMSE), mean absolute error (ME) and correlation index $(r)$. Results showed that Model 1 had a better performance, presenting a correlation index of 0.77 and RMSQ e ME less than $1.8 \mathrm{Mj}$. This indicates that this model is usable for solar radiation estimation, but given the simplicity, performance and significance.

Keywords: Solar radiation; Climatic data; Air temperature; Solar radiation estimation.

\section{Introdução}

A busca pela diversificação das fontes de energia associada a crescente preocupação com a preservação do meio ambiente e o aumento constante na demanda energética tem sido tema amplamente discutido em vários segmentos do meio acadêmico.

Em processo de ampla expansão, as fontes renováveis vêm se tornando cada vez mais competitivas devido à redução dos custos em função do ganho de escala e dos avanços tecnológicos. Dentre essas fontes a solar tem se mostrado uma ótima alternativa para produção de energia elétrica. Além disso, a energia gerada a partir da radiação solar gera menor impacto ambiental e constitui-se como uma fonte gratuita e abundante de energia, com baixos requisitos de manutenção (Souza et al., 2016; Furlan et al., 2012; Ehnberg e Bollen, 2005).

Segundo a ANEEL (2005), o aproveitamento da energia solar pode ser realizado diretamente para iluminação, aquecimento de fluidos e ambientes ou ainda para geração de potência mecânica ou elétrica, como fonte de energia térmica. A energia solar pode ainda ser convertida diretamente em energia elétrica por meio de efeitos sobre materiais, dentre os quais 0 termoelétrico e fotovoltaico.

De acordo com Nascimento (2017), o Brasil possui expressivo potencial para geração de energia elétrica a partir de fonte solar, contando com níveis de irradiação solar superiores aos de países onde projetos para aproveitamento de energia solar são amplamente disseminados, como Alemanha, França e Espanha. Apesar disso, o uso da fonte para geração de energia elétrica não apresenta a mesma relevância que possui em outros países, nem o mesmo desenvolvimento de outras fontes renováveis, como eólica e biomassa, que já representam, respectivamente, $6,7 \%$ e $9,4 \%$ da capacidade de geração instalada no Brasil, contra apenas $0,05 \%$ da fonte solar.

A determinação e o aproveitamento eficaz do potencial energético solar estão atrelados ao conhecimento das variações espaciais e da evolução sazonal da radiação solar incidente na superfície. No entanto, existem vários fatores que dificultam a obtenção dessas informações. Isto porque são poucas as estações meteorológicas que dispõem de equipamentos para medição da radiação solar global (Buriol et al., 2012).

De acordo com Souza et al. (2016) este problema pode estar associada ao elevado custo instrumental e a frequente necessidade de manutenções pelas quais precisam passar, o que limita e restringe as medidas de rotina em centros de pesquisa e universidades (Coppolino, 1994; Ampratwum e Dorvlo, 1999), afetando, consequentemente, a obtenção destas informações em extensão 
continental (Souza et al., 2011). Tudo isso acaba por dificultar ainda mais a implantação e operação de uma rede de estações solarimétricas.

Para tentar driblar a falta de dados medidos de radiação solar em função da não existência de uma rede de medição mais abrangente, modelos matemáticos foram desenvolvidos $\mathrm{e}$ calibrados para estimar a radiação em diferentes partes do mundo, a saber, Silva et al. (2012), Santos (2014) e Souza et al. (2017) para o Brasil, Saffaripour (2013) para o Irã, Bajpai (2009) para a Índia, Chegaar (1998) para Argélia/ Espanha, Li (2014) para China, Datta e Datta (2013) para Bangladesh, Meza e Varas (2000) para o Chile, Allen (1997) para os EUA, e Umoh (2013) para a Nigéria. Esses modelos estimam a radiação solar global em função de parâmetros meteorológicos como temperatura do ar, pressão atmosférica, umidade relativa, número de horas de brilho solar, velocidade do vento, cobertura de nuvens e chuva.

Os modelos empíricos mínimos requerem uma entrada de apenas um parâmetro meteorológico para estimar a radiação solar e incluem modelos baseados, por exemplo, na relação entre a insolação astronômica efetiva e o fotoperíodo (Angstrom, 1924), na temperatura do ar (Bristow e Campbell, 1984; Hargreaves, 1994; Allen, 1997), e na cobertura de nuvem (Santos, 2014; Kostić e Mikulovic, 2017). Existem também modelos intermediários que requerem a entrada de dois ou mais parâmetros meteorológicos (Okundamiya et al., 2016).

O conhecimento da variabilidade da radiação solar global de um determinado local é base para muitos estudos que encontram aplicações em diversas áreas, tais como meteorologia, climatologia, agricultura, engenharia, e no planejamento e aproveitamento da energia solar.

Diante disso e do crescente interesse no aproveitamento da energia solar em Mato Grosso do Sul, o objetivo deste trabalho é avaliar a estimativa da radiação solar global para o estado sul mato-grossense a partir de dois modelos diferentes. 0 primeiro modelo considera que a radiação solar média mensal é uma função linear da temperatura média mensal do ar e o segundo é o modelo clássico de Hargreaves e Samani (1982) que assume que a radiação solar é uma função da diferença entre a temperatura máxima e mínima diária do ar, partindo do pressuposto de que essa diferença geralmente indica nebulosidade diária. E, adicionalmente, com base nos resultados alcançados, traçar isolinhas de radiação solar global para todo o estado a partir do modelo que tenha apresentado melhor desempenho.

\section{Materiais e métodos}

\section{Área de estudo}

A área de estudo é o Estado de Mato Grosso do Sul que está localizado no sul da Região Centro-Oeste do Brasil, entre os paralelos $18^{\circ} \mathrm{S}-23^{\circ} \mathrm{S}$ e os meridianos $52^{\circ} \mathrm{W}-57,5^{\circ} \mathrm{W}$.

Para a realização deste estudo foram utilizados dados da temperatura do ar e da radiação solar global de janeiro a dezembro de 2017, obtidos em estações meteorológicas automáticas pertencentes ao Instituto Nacional de Meteorologia (INMET), disponíveis no endereço eletrônico da própria instituição (http://www.inmet.gov.br). A localização das estações meteorológicas para as quais os estudos de calibração e desempenho dos modelos foram realizados é ilustrada na Figura 1.

\section{Dados climatológicos}

$\mathrm{Na}$ maior parte do território do estado sul-mato-grossense predomina o clima do tipo tropical ou tropical de altitude, com chuvas de verão e inverno seco, caracterizado por médias termométricas que variam entre $25^{\circ} \mathrm{C}$ na Baixada do Paraguai e $24{ }^{\circ} \mathrm{C}$ no planalto. No extremo meridional ocorre o clima subtropical, em virtude de uma latitude um pouco mais elevada e do relevo de planalto. 


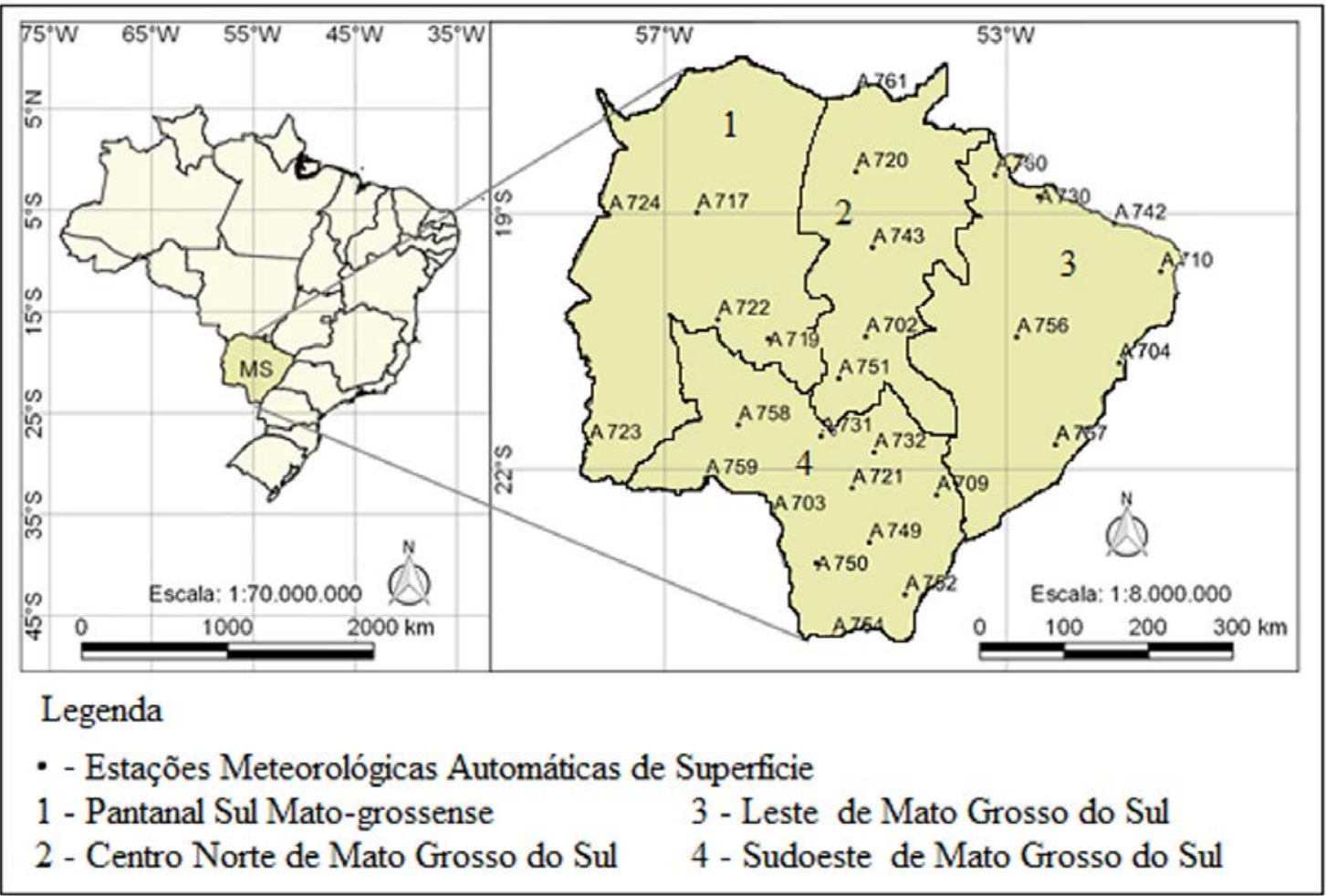

Figura 1. Localização das estações meteorológicas automatizadas utilizadas no experimento bem como as mesorregiões do estado de Mato Grosso do Sul, Brasil.

Os valores das temperaturas médias mensais e anuais registrados levam ao entendimento de que a variação espacial e sazonal desta variável climatológica segue as características da região, sendo a altitude e a continentalidade, assim como a ação das massas Tropical Atlântica, Tropical Continental e Polar Atlântica as responsáveis pelas principais variações observadas.

As maiores médias térmicas são observadas entre os meses de outubro e março, que correspondem ao verão no domínio dos climas tropicais no Hemisfério Sul, sendo o mês de outubro o que apresenta as maiores médias, visto que este se caracteriza pela transição entre o período seco e chuvoso. Assim, as mudanças nos padrões de circulação atmosférica, os altos índices de evapotranspiração, as baixas velocidades dos ventos, as precipitações incipientes e a baixa umidade do ar, favorecem a elevação das temperaturas, que indicam o início do verão. Outra análise que pode ser feita a partir das temperaturas médias é que a amplitude térmica observada entre os meses com maiores e menores temperaturas varia em torno de $11{ }^{\circ} \mathrm{C}$ em média, entre o mês de junho (menores médias térmicas) e o mês de outubro (mês mais quente).

As geadas são comuns no sul do estado, registrando em média três ocorrências do fenômeno por ano. Observa-se o mesmo regime de chuvas de verão e inverno seco e que a pluviosidade anual é, também, de aproximadamente $1.500 \mathrm{~mm}$.

Em razão da baixa influência que a topografia regional exerce sobre a pluviosidade, a precipitação anual apresenta uma distribuição muito simples: um núcleo mais chuvoso ao norte de Mato Grosso do Sul, com 1.492 $\mathrm{mm}$, decrescendo para leste e oeste; no extremo oeste do estado, o regime cai 
para $1.117 \mathrm{~mm}$; a região sul apresenta os maiores valores, $1.660 \mathrm{~mm}$.

\section{Modelos de estimativa de radiação solar}

A radiação solar que atinge a superfície terrestre é composta pela radiação difusa decorrente dos processos de espalhamento que a radiação solar sofre na atmosfera e pela radiação direta que atinge a superfície diretamente. A soma das duas componentes resulta na radiação global. É esta radiação que se quer estimar.
Os modelos baseados na temperatura do ar para estimar a radiação solar são úteis para quantificar os níveis de radiação solar em diferentes locais ao redor do mundo. Assim, para estimativa de radiação solar global $\left(Q_{g}\right)$ fez-se uso de dois modelos diferentes. Ambos baseados na temperatura do ar, considerando que esta é uma das variáveis climatológicas mais comumente disponíveis. 0 primeiro modelo para prever a radiação solar global é expresso por:

$$
Q_{g}=m_{1} \cdot T_{m}+c_{1}
$$

onde $T_{\mathrm{m}}=\left(T_{\max }-T_{\min }\right) / 2$, é a temperatura média diária, e $T_{\max }$ e $T_{\min }$ são, respectivamente, as médias diárias máxima e mínima da temperatura do ar $\left({ }^{\circ} \mathrm{C}\right)$ por um período de um mês, $m_{1}$ e $c_{1}$ são constantes empíricas.

Tais constantes foram determinadas utilizando as médias mensais medidas da radiação solar e da temperatura do ar. A relação expressa na Eq. (1) é motivada pela observação, de que a radiação solar global medida é proporcional às temperaturas médias do ar observadas ao longo do ano. De acordo com Dlamini et al. (2017), estudos anteriores consideraram equações lineares semelhantes para prever a radiação solar, como é o caso, por exemplo, do modelo clássico de Angstrom (Angstrom, 1924) que pressupõe que a magnitude da radiação solar é proporcional à duração do período diurno (Angstrom, 1924; Meza, 2000; Liu et al., 2012; Yakubu, 2012).

Segundo Liu et al (2012), a maioria dos modelos baseados em temperatura do ar encontrados na literatura, assumem que a radiação solar global é uma função da diferença entre a temperatura diária máxima e mínima do ar, uma vez que o ciclo diurno da temperatura reflete a variação da radiação ao longo do dia. Tipicamente, a menor temperatura ocorre próximo ao nascer do sol, como resultado de uma noite de resfriamento radiativo da superfície da Terra. A temperatura mais alta ocorre usualmente no começo ou meio da tarde, enquanto o pico de radiação ocorre ao meio dia (Grimm, 1999).

Os resultados obtidos com a Eq. (1) foram então comparados com o modelo clássico de Hargreaves e Samani (1982), que pressupõe que a radiação solar global pode ser estimada a partir da seguinte equação:

$$
Q_{g}=m_{2} Q_{0} \sqrt{T_{\max }-T_{\min }}
$$

onde $Q_{0}$ é a radiação extraterrestre e $m_{2}$ é uma constante empírica que varia de acordo com o clima da localidade analisada (Allen, 1997). 
A radiação solar extraterrestre $\left(Q_{0}\right)$ é uma função da latitude $(\phi)$ e pode ser facilmente avaliada ou obtida na literatura (Duffie e Beckman, 2013). No caso desse estudo, a radiação extraterrestre foi estimada para cada localidade a partir da seguinte equação:

$$
Q_{0}=37,60 \cdot(\bar{D} / D)^{2} \cdot(H \cdot \operatorname{sen} \varphi \cdot \operatorname{sen} \delta+\cos \varphi \cdot \cos \delta \cdot \operatorname{sen} H)
$$

onde o fator $(\bar{D} / D)^{2}$ expressa a variação da densidade de fluxo de radiação que atinge a superfície normal aos raios, no topo da atmosfera, em virtude da distância variável da Terra em relação ao Sol; $\delta$ é a declinação e $H$ o ângulo horário (Vianello e Alves, 2004).

A radiação solar que atinge a superfície terrestre é composta pela radiação difusa decorrente dos processos de espalhamento que a radiação solar sofre na atmosfera e pela radiação direta que atinge a superfície diretamente. A soma das duas componentes resulta na radiação global. Assim, a radiação solar na superfície terrestre $Q_{g}$ é sempre inferior a extraterreste $Q_{0}$, uma vez que ao passar pela atmosfera terrestre $Q_{g}$ é modificada por processos de dispersão e absorção devido à presença de nuvens e partículas atmosféricas.

Para efeito didático os modelos representados pelas equações (1) e (2), serão doravante chamados, respectivamente, Modelo 1 e Modelo 2.

Avaliação dos parâmetros do modelo. As constantes empíricas para os Modelos 1 e 2 foram avaliadas usando os dados observados da radiação solar média mensal e da temperatura mensal em Campo Grande durante o ano de 2017. Esses parâmetros foram calculados usando o algoritmo LevenbergMarquardt para ajuste de curva. O algoritmo de Levenberg-Marquardt busca numericamente os parâmetros que ajustam pelo método dos mínimos quadrados uma dada função genérica (em geral não linear) a um dado conjunto de ponto (Lima, 2009). Nas Figuras 2a e $2 \mathrm{~b}$ são mostrados graficamente para os Modelos 1 e 2, respectivamente, os resultados da curva para Campo Grande. Neste caso, os melhores ajustes foram descritos por $m_{1}=1,12 \pm 0,08$ e $_{1}=-8,98$ \pm 1 para o Modelo 1 e $m_{2}=0,21 \pm 0,002$ para o Modelo 2.

Para locais com uma ligeira variação das condições climáticas com relação às de Campo Grande, os mesmos parâmetros dos modelos podem ser aplicados para prever os níveis de radiação solar. No entanto, para as localidades que tem uma variação mais ampla nas condições climáticas é necessário ajustar corretamente os parâmetros dos modelos.

Coeficientes de calibração e avaliação de desempenho. No período analisado, um ano foi utilizado para calibrar os coeficientes para avaliar o desempenho dos modelos. Obtiveram-se os coeficientes dos modelos a partir do método dos mínimos quadrados da diferença entre os valores observados e os estimados. 


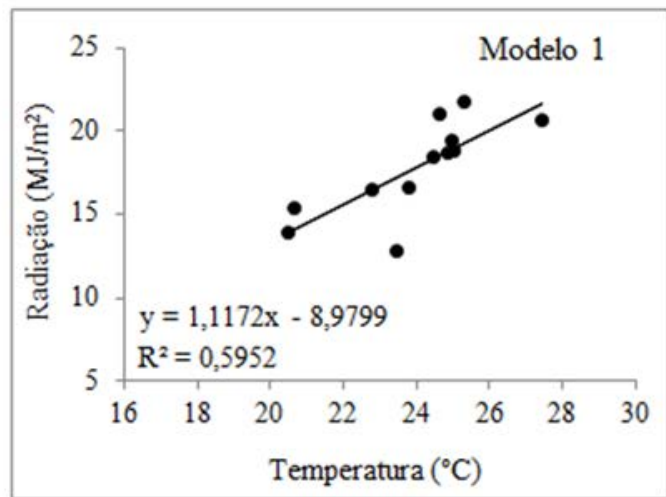

(a)

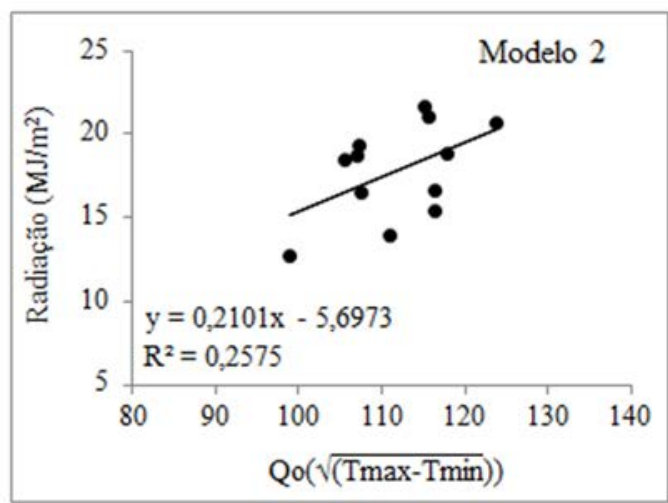

(b)

Figura 2. Avaliação das constantes empíricas para os dois modelos: (a) Radiação solar média mensal contra temperaturas médias mensais de 2017 para Campo Grande (Modelo 1). (b) Radiação solar média mensal contra produto entre a radiação extraterrestre e a raiz quadrada das diferenças entre as temperaturas médias mensais máxima e mínima de 2017 para Campo Grande (Modelo 2).

Para avaliar o desempenho de cada modelo de maneira similar aos trabalhos de avaliação de modelos de estimativa de $Q_{0}$ foram utilizados os seguintes índices estatísticos: o Viés, o (Eq. 4), o erro quadrático médio (EQM) (Eq. 5), o erro absoluto médio (EA) (Eq. 6) e o índice de Correlação (r) (Eq. 7):

$$
\begin{aligned}
& \text { Viés }=\frac{1}{n} \sum_{i=1}^{n}\left(E_{i}-O_{i}\right) \\
& E Q M=\left[\frac{1}{n} \sum_{i=1}^{n}\left(E_{i}-O_{i}\right)^{2}\right]^{0,5} \\
& E A=\frac{1}{n} \sum_{i=1}^{n}\left|E_{i}-O_{i}\right| \\
& r=\frac{\sum\left(E_{i}-\bar{E}_{i}\right)\left(O_{i}-\bar{O}\right)}{\sqrt{\left[\sum\left(E_{i}-\bar{E}_{i}\right)^{2}\right]\left[\sum\left(O_{i}-\bar{O}\right)^{2}\right]}}
\end{aligned}
$$

em que: $E_{i}$ - valor estimado; $O_{i}$ - valor observado; $\bar{O}$ - média dos valores observados; $n$ - número de observações.

Esta correlação pode variar de muito fraca a muito forte de acordo com os intervalos de $r$ contidos na Tabela 1 (Devore, 2006). No entanto, antes de tirar qualquer conclusão sobre os valores dos coeficientes de correlação é necessária a aplicação de um teste estatístico para conhecer o grau real de ligação entre as variáveis analisadas (Oliveira e Souza, 2017; Lima, 2011). 
Tabela 1.Coeficiente de correlação de Pearson.

\begin{tabular}{l|l}
\hline Intervalos & Definição \\
\hline 0,00 a 0,19 & Correlação bem fraca \\
0,20 a 0,39 & Correlação fraca \\
0,40 a 0,69 & Correlação moderada \\
0,70 a 0,89 & Correlação forte \\
0,90 a 1,00 & Correlação bem forte \\
\hline
\end{tabular}

Fonte: Devore (2006).

Neste estudo, o teste estatístico utilizado foi o teste de significância $t$ de

$$
t=\frac{r \sqrt{n-2}}{\sqrt{1-r^{2}}}
$$

O coeficiente de correlação crítico $\left(r_{c}\right)$, ou seja, valor para o qual se aceita ou
Student, com os seguintes valores do parâmetro $t$ :

$$
r_{c}=\sqrt{\frac{t^{2}}{(n-2)+t^{2}}}
$$

não a hipótese estatística, pode ser obtido a partir da Eq. (8) e dado por:
As séries de dados utilizadas foram de 28, 30 e 31 dias. Para a correlação desses dados, com 2 graus de liberdade, tem-se n - 28 =26, n-30 = 28 e $n-31=29$. Com base nessa informação e utilizando os valores tabelados de $t$, os coeficientes de correlação crítico $r_{c}$ calculados estão na Tabela 2.

Tabela 2 - Coeficiente de correlação crítico.

\begin{tabular}{c|ccccccc}
\hline Significância & \multicolumn{2}{|c|}{$\mathbf{9 0 \%}$} & \multicolumn{2}{c|}{$\mathbf{9 5 \%}$} & \multicolumn{2}{c}{$\mathbf{9 9 \%}$} \\
\hline (n-2) & $\boldsymbol{t}^{\mathbf{1}}$ & $\boldsymbol{r}_{\boldsymbol{c}}$ & $\boldsymbol{t}^{\mathbf{1}}$ & $\boldsymbol{r}_{\boldsymbol{c}}$ & $\boldsymbol{t}^{\mathbf{1}}$ & $\boldsymbol{r}_{\boldsymbol{c}}$ \\
\hline 26 & 1,315 & 0,25 & 1,706 & 0,32 & 2,479 & 0,44 \\
28 & 1,313 & 0,24 & 1,701 & 0,31 & 2,467 & 0,42 \\
29 & 1,311 & 0,24 & 1,699 & 0,30 & 2,462 & 0,42 \\
\hline
\end{tabular}

${ }^{1}$ Fonte: Barbetta (2010).

Caso o coeficiente de correlação $r$ calculado seja igual ou superior ao valor encontrado com o valor do $t$ crítico para um determinado grau de liberdade e percentual de significância, a hipótese de nulidade é rejeitada e a tendência observada é verdadeira para aquele nível de significância obtido (Bruni, 2007; Menezes, 2010; Lima, 2011; Oliveira e Souza, 2017). 
Estimativas de radiações solar

Para a estimativa da distribuição espacial da radiação solar ao longo do estado de Mato Grosso do Sul utilizou-se o método de interpolação de dados. Mapas de isolinhas de distribuição espacial de Radiação Solar foram plotadas utilizando o software QGIS 2.14 (QGIS Development Team, 2014). Para o modelo que apresentou melhor desempenho foi elaborado um mapa de isolinhas, cuja variação temporal foi o período mensal.

0 método de interpolação adotado foi o IDW (Inverse Distance Weighting), que segundo Silva (2015) é um dos métodos mais utilizados em análises georreferenciadas. O IDW, proposto por Shepard (1968), é um método determinístico utilizado para estimativa ou interpolação de dados e pressupõe que quanto maior a distância do local observado estiver ponto estimado, menor será sua influência sobre o valor interpolado. Assim, o IDW assume que cada ponto amostral possui influência sobre o ponto interpolado e que essa influência diminui com o aumento da distância (Luo et al., 2008).

\section{Resultados e discussão}

\section{Desempenho dos modelos contra dados observados}

Os parâmetros de valores calculados foram utilizados para estimar os níveis de radiação solar global para o ano de 2017. Os valores estimados foram então comparados aos valores observados. A Figura 3a mostra uma amostra desses resultados. Nota-se que os dados estimados com o Modelo 1 são mais consistentes que os estimados com o Modelo 2 em comparação aos dados observados. Nesta Figura é mostrada a curva da radiação solar extraterrestre $\left(Q_{0}\right)$ em uma superfície horizontal para Campo Grande, além dos valores de radiação observados e estimados. Adicionalmente, é mostrada também a relação entre a variação da temperatura e da radiação solar (Figura $3 b$ ).

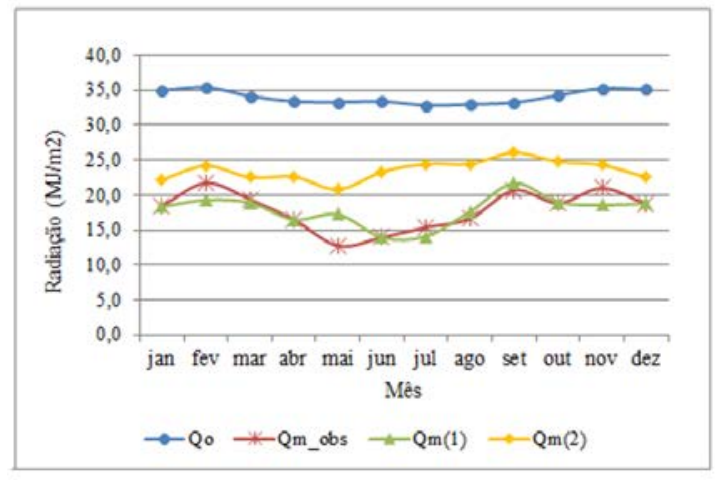

(a)

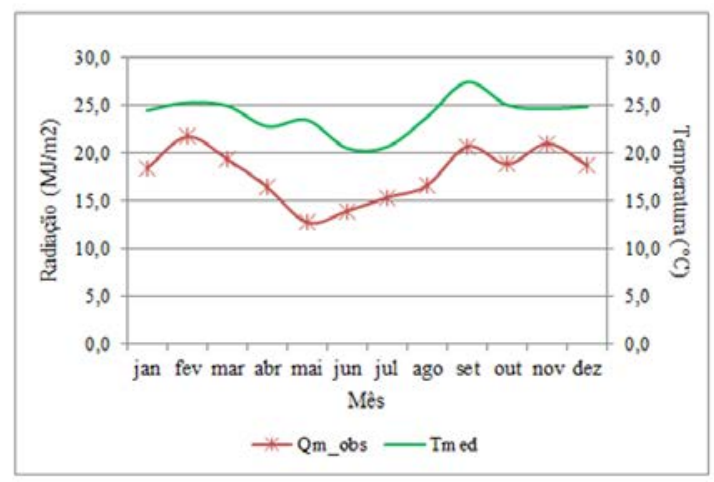

(b)

Figura 3. (a) Radiação solar média mensal observada e estimada para Campo Grande. (b) Variação da temperatura e da radiação solar ao longo do ano.

Na Tabela 3 têm-se os valores da radiação global medida $\left(Q_{g}\right)$, das radiações estimadas com o Modelo 1 $\left[Q_{g}(1)\right]$ e com o Modelo $2\left[Q_{g}(2)\right]$ e do Viés obtidos a partir da análise feita para
Campo Grande. Estas são medidas fundamentais de precisão nos cálculos de energia solar (Saffaripour, 2013, Li et al., 2014; Okundamiya, 2016). 
Analisando os resultados do Viés, nota-se que o Modelo 1 superestimou a radiação global em 6 dos 12 meses analisados, sendo o maior valor obtido para o mês de maio $(4,48)$ e o menor para junho $(-0,01)$. Já o Modelo 2 superestimou a radiação observada em todos os meses, com destaque para os meses de maio a agosto que apresentaram as maiores diferenças (acima de 7,8). Esses resultados são notáveis quando se observa a Figura 3a, que mostra radiação solar média mensal observada e estimada para Campo Grande.

Tabela 3. Análise da diferença entre a radiação solar medida e estimada para o ano de 2017 em Campo Grande, MS.

\begin{tabular}{l|ccc|cc}
\hline \multirow{2}{*}{ Meses } & \multicolumn{3}{|c|}{ Radiação solar média } & \multicolumn{2}{c}{ Viés } \\
\cline { 2 - 5 } & $\boldsymbol{Q}_{g}(\mathbf{o b s})$ & $\boldsymbol{Q}_{g}(\mathbf{1})$ & $\boldsymbol{Q}_{g}(\mathbf{2})$ & $\boldsymbol{Q}_{g}(\mathbf{1})$ & $\boldsymbol{Q}_{g}(\mathbf{2})$ \\
\hline Jan & 18,41 & 18,33 & 22,17 & $-0,08$ & 3,76 \\
Mev & 21,71 & 19,27 & 24,18 & $-2,44$ & 2,47 \\
Abr & 19,37 & 18,89 & 22,52 & $-0,48$ & 3,15 \\
Maio & 16,44 & 16,47 & 22,61 & 0,02 & 6,17 \\
Jun & 12,73 & 17,21 & 20,80 & 4,48 & 8,07 \\
Jul & 13,92 & 13,91 & 23,31 & $-0,01$ & 9,38 \\
Ago & 15,36 & 14,09 & 24,46 & $-1,27$ & 9,10 \\
Set & 16,63 & 17,61 & 24,45 & 0,98 & 7,82 \\
Out & 20,69 & 21,68 & 26,03 & 0,98 & 5,34 \\
Nov & 18,83 & 18,96 & 24,77 & 0,13 & 5,93 \\
Dez & 20,98 & 18,56 & 24,28 & $-2,43$ & 3,30 \\
Média & 18,68 & 18,77 & 22,51 & 0,09 & 3,83 \\
\hline
\end{tabular}

Em estudos que envolvem estimativa de dados é comum querer saber se há relação entre os dados estimados e os observados. 0 método usualmente conhecido para medir a correlação entre tais dados é o Coeficiente de Correlação Linear de Pearson $(r)$.

Tomando como base os dados analisados para Campo Grande, tem-se que os coeficientes de correlação encontrados para os Modelo 1 e 2 foram de 0,77 e 0,51 , respectivamente. Tais resultados revelam, de acordo com a classificação de Devore (2006), uma forte correlação entre a radiação estimada com o Modelo 1 e a observada, enquanto a radiação estimada com o Modelo 2 apresentam uma correlação moderada.

Para verificar se as correlações obtidas entre os dados de radiação observados e estimados são significativas, utilizou-se o teste de $t$ de Student. Com base nos valores de $t$ e do coeficiente de correlação crítico $\left(r_{c}\right)$ contidos na Tabela 2 , tem-se que os coeficientes de correlação $(r)$ encontrados são superiores aos valores de $r_{c}$ para o nível de significância de $99 \%$. Portanto, pode-se concluir que há evidências estatísticas suficientes para afirmar que as séries de dados observados e as séries de dados estimados pelos dois modelos, estão bem correlacionadas.

No que diz respeito aos erros absoluto médio (EA) e quadrático médio (EQM), apresentados na Tabela 4, verifica-se que o Modelo 1 obteve os melhores resultados $(E A=1,12 \mathrm{~m} / \mathrm{s}$ e $\mathrm{EQM}=1,73$ ), tendo $\mathrm{o}$ Modelo 2 apresentados valores superiores a 5 
tanto para o EA como para o EQM. Logo, esses resultados indicam um melhor desempenho do Modelo 1 em relação ao
Modelo 2 em estimar a radiação solar global.

Tabela 4. Índices estatísticos obtidos para as séries de dados estimados e observados de radiação solar para o ano de 2017 em Campo Grande, MS.

\begin{tabular}{c|ccc}
\hline \multirow{2}{*}{ Modelos } & \multicolumn{3}{|c}{ Índices estatísticos } \\
\cline { 2 - 4 } & $\mathbf{E M}$ & $\mathbf{E Q M}$ & $\mathbf{r}$ \\
\hline $\mathbf{1}$ & 1,12 & 1,73 & 0,77 \\
$\mathbf{2}$ & 5,69 & 6,15 & 0,51 \\
\hline
\end{tabular}

\section{Estimativas de radiações solares em outros locais}

Diante dos resultados obtidos para Campo Grande, optou-se por utilizar o Modelo 1 para estimar a radiação solar ao longo do estado sul-mato-grossense. A partir dos dados obtidos para Campo Grande com o Modelos 1 foi possível calibrar os coeficientes e realizar testes para várias localidades sul-matogrossenses que possuem estações meteorológicas automatizadas (Figura 1). Após os testes foram considerados os resultados que apresentaram EA menores ou iguais a $1,12 \mathrm{MJ} / \mathrm{m}^{2}$ e coeficientes de correlações superiores a 0,70 . As estimativas da radiação para o ano de 2017 em outras localidades são mostradas na Figura 4.

A radiação solar mensal estimada para o estado de Mato Grosso do Sul apresentou variações de 11 a $24 \mathrm{MJ} / \mathrm{m}^{2}$, com média de $17,5 \mathrm{MJ} / \mathrm{m}^{2}$. Percebe-se que de setembro a março, meses que correspondem às estações da primavera e do verão, foram observados os maiores níveis de radiação solar, com destaque para os o mês de setembro e fevereiro que obtiveram os maiores níveis de radiação, coincidindo com os meses que registraram os níveis mais elevados de radiação solar no estado. Em contrapartida, para os meses correspondentes às estações do outono e do inverno, ou seja, de abril a agosto, os níveis de radiação solar foram menores, sendo os níveis mais baixos de radiação verificados para os meses de junho e julho, que correspondem à época mais fria do ano no estado. Neste contexto, os maiores níveis de radiação solar verificados podem ser atribuídos a maior duração do dia, que no verão aumenta conforme se observam latitudes maiores (Silva, 2001).

0 resultado do mês de setembro mostra uma distribuição isotrópica em torno do relevo, numa ilustração da distribuição esperada em condições de declinação local nula. Nesta condição, a relação esperada com o relevo em períodos diurnos a anuais é uma função aproximada do cosseno da declividade. Portanto, espera-se que os maiores níveis de radiação solar ocorram nas áreas planas e que sejam reduzidas com a declividade (área do pantanal). Em condições pouco diferentes, observa-se no mês de dezembro (que corresponde ao período de menor declinação local na área do estudo) índices de radiação solar distribuídos de forma mais uniforme em relação às orientações de vertente, e áreas planas associadas aos maiores níveis.

Em contraposição, no mês de junho (período de maior declinação local) o efeito da orientação de vertentes sobre a distribuição da radiação solar foi mais evidente, o que pode ser verificado pelo forte contraste entre as vertentes nas orientações Norte e Sul. Na área do pantanal, a declinação solar não atinge a latitude local, portanto este efeito se 
expressa em todos os períodos. Nas situações de alta declinação local, devese creditar a intensidade do efeito da orientação de vertentes em parte à baixa elevação geral do Sol ao longo de sua trajetória diurna, o que promove maior variação momentânea dos ângulos de incidência dos raios solares no terreno, favorecendo também o sombreamento. Porém, a alta declinação local está também associada a uma distribuição mais restrita dos ângulos azimutais solares em torno da direção do Equador (ao Norte, para o hemisfério Sul). Essa prevalência em azimute resulta no controle da radiação solar pela orientação de vertentes. Em latitudes além dos trópicos, ocorre em qualquer período de integração ao longo do ano.

A amplitude de variação se mostrou crescente de acordo com as classes de declividade, como verificado também por Buffo et al. (1972) e Kumar et al. (1997). Os níveis máximos de radiação solar anual incidente no terreno apresentaram aumento discreto em comparação com a forte diminuição dos níveis mínimos. Adicionalmente, a amplitude da radiação solar anual mostrou-se relacionada à latitude. Conforme se observou em relação à condição de pequena declinação local, em que as variações estão relacionadas mais ao cosseno da declividade do que às orientações de vertentes, amplitude absoluta de radiação solar anual apresentou-se baixa e relativamente estável.

A análise temporal, topográfica, geográfica e atmosférica do cálculo e distribuição da radiação solar no terreno permitiu avaliar o potencial de variação da radiação sob diversas condições. Dada a complexidade das interações entre posição (latitude), atmosfera, período e o próprio relevo na distribuição da radiação solar incidente, a observação isolada de cada fator numa determinada situação é pouco passível de extensão às demais circunstâncias demandadas pelas possíveis aplicações.
Vale salientar que para períodos semianuais ou mais curtos, deve-se considerar a diferença entre a latitude e a declinação solar representativa do período. Quanto maior este ângulo, mais intenso o efeito do relevo e maior o peso da orientação de vertentes nas variações de radiação solar. Ao contrário, quando a declinação média do período analisado coincide com a latitude local, o efeito da orientação de vertentes, assim como do relevo em geral, é reduzido, restando uma pequena variação em função da declividade, que pode ocasionar maiores níveis de radiação solar nas áreas planas.

\section{Conclusões}

Os resultados obtidos indicam que, na ausência de dados observados de radiação solar incidente, estimativas podem ser feitas a partir dos dados da temperatura máxima e mínima do ar usando modelos empíricos.

A radiação solar estimada com o Modelo 1 mostrou-se fortemente correlacionada com a radiação observada para o período estudado. Além disso, a pequena diferença entre os dados estimados com o Modelo 1 e os dados observados de radiação solar para o estado de Mato Grosso do Sul e a proximidade com resultados encontrados na literatura, indica que este modelo pode ser empregado para estimar a radiação solar global.

Ao longo da última década houve um crescente interesse no aproveitamento da energia solar em Mato Grosso do Sul, principalmente usando módulos fotovoltaicos para reduzir a quantidade de energia importada dos países vizinhos. Acreditase que estimativa da radiação solar usando dados de temperatura do ar, será de grande utilidade na prospecção de regiões com potencial para exploração de energia solar que não dispõem de equipamentos de medição de radiação, e, portanto, ajudará no desenvolvimento da indústria de energia solar no país. 


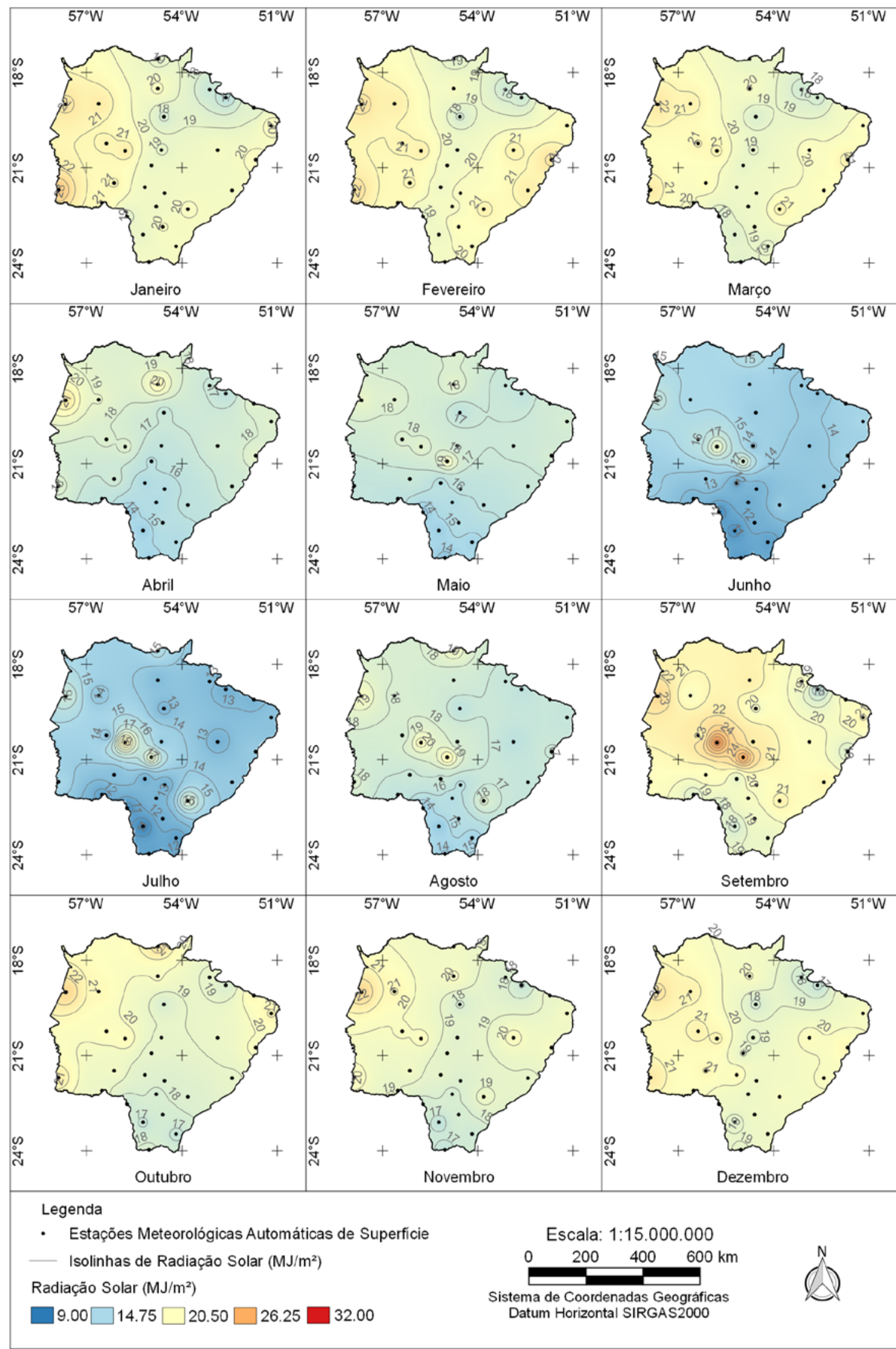

Figura 4. Distribuição espacial e temporal da estimativa de radiação solar global a partir do Modelo 1, usando a temperatura média do ar para o estado de Mato Grosso do Sul. 


\section{Conflitos de interesse}

Os autores declaram não haver conflitos de interesse.

\section{Referências}

Allen, R. G. Self-calibrating method for estimating solar radiation from air temperature. Journal of Hydrologic Engineering, v. 2, n. 2, p.56-67, 1977. https://doi.org/10.1061/(ASCE)10840699(1997)2:2(56)

Ampratwum, D. B.; Dorvlo, A. S. S. Estimation of solar radiation from the number of sunshine hours. Applied Energy, v. 63, n. 3, p. 161-167, 1999. https://doi.org/10.1016/ S0306-2619(99)00025-2

ANEEL - Agência Nacional de Energia Elétrica. Atlas da energia elétrica do Brasil. 2. ed. Brasília: ANEEL, 2005. Disponível em: <http://www.aneel.gov.br/documents/6568 35/14876406/2005_AtlasEnergiaEletricaBra sil2ed/06b7ec52-e2de-48e7-f8be-

1a39c785fc8b>. Acesso em: 29 out. 2018.

Angstrom, A. Solar and terrestrial radiation. Quarterly Journal of the Royal Meteorological Society, v. 50, p. 121-126, 1924. https://doi.org/10.1002/qj.497050 21008

Bajpai, U.; Singh K. Estimation of instant solar radiation by using instant temperature. Acta Montanistica Slovaca, v. 14, n. 2, p. 189-196, 2009.

Barbetta, P. A. Estatística aplicada às Ciências Sociais. 7. ed. Florianópolis: Editora da UFSC, 2010.

Bristow, K. L.; Campbell, G. S. On the relationship between incoming solar radiation and daily maximum and minimum temperature. Agricultural and Forest Meteorology, v. 31, n. 2, p. 159-166, 1984. https://doi.org/10.1016/0168-1923(84)90 017-0

Bruni, A. L. Estatística aplicada à gestão empresarial. São Paulo: Atlas, 2007.

Buffo, J.; Fritschen, L. J.; Murphy, J. L. Direct solar radiation on various slopes from 0 to 60 degrees North latitude. Portland: Pacific Northwest Forest and Range Experiment Station, 1972. Disponível em: <http://www.fs.fed.us/pnw/pubs/pnw_rp14 2.pdf>. Acesso em: 29 out. 2018.
Buriol, G. A.; Estefanel, V.; Heldwein, A. B.; Prestes, S. D.; Horn, J. F. C. Estimativa da radiação solar global a partir dos dados de insolação, para Santa Maria-RS. Ciência Rural, v. 42, n. 9, p. 1563-1567, 2012. https://doi.org/10.1590/S0103-84782012 005000059

Chegaar, M.; Lamri, A.; Chibani, A Estimating global solar radiation using sunshine hours. Revue Energies Renouvelables: Physique Energétique, n. especial 4, p. 7-11, 1998. Disponível em: <https://www.cder.dz/ download/sipe_2.pdf>. Acesso em: 29 out. 2018.

Coppolino, S. A new correlation between clearness index and relative sunshine. Renewable Energy, v. 4, n. 4, p. 417-423, 1994. https://doi.org/10.1016/0960-1481 (94)90049-3

Datta, D.; Datta, B. K. Empirical model for the estimation of global solar radiation in Dhaka, Bangladesh. International Journal of Research in Engineering and Technology, v. 2 , n. 11 , p. $649-653$, 2013. https://doi.org/ 10.15623/ijret.2013.0211099

Devore, J. L. Probabilidade e estatística para Engenharia e Ciência. São Paulo: Thomson Pioneira, 2006.

Duffie, J.A.; Beckman, W. A. Solar engineering of thermal processes. New Jersey: John Wiley, 2013.

Ehnberg, J. S. G.; Bollen, M. H. J. Simulation of global solar radiation based on cloud observations. Solar Energy, v. 78, n. 2, p. 157-162, 2005. https://doi.org/10.1016/ j.solener.2004.08.016

Furlan, C.; Oliveira, A. P.; Soares, J.; Codado, G.; Escobedo, J. F. The role of clouds in improving the regression model for hourly values of diffuse solar radiation. Applied Energy, v. $92, \quad$ p. $240-254,2012$. https://doi.org/10.1016/j.apenergy.2011.10. 032

Grimm, A. M. Controles da temperatura. Notas de aula, 1999. Disponível em: <https://fisica.ufpr.br/grimm/aposmeteo/ca p3/cap3-5.html>. Acesso em: 28 out 2018.

Hargreaves, G. H.; Samani, Z. A. Estimating potential evapotranspiration. Journal of the Irrigation and Drainage Division, v. 108, n. 3, p. 223-230, 1982.

Hargreaves, G. H. Simplified coefficients for estimating monthly solar radiation in 
North America and Europe. Logan, Utah: Utah State University, 1994.

Kostić, R.; Mikulovic, J. The empirical models for estimating solar insolation in Serbia by using meteorological data on cloudiness. Renew Energy, v. 114, Part B, p. 1281-1293, 2017. https://doi.org/10.1016/j.renene. 2017.08.013

Kumar, L.; Skidmore, A. K.; Knowles, E. Modelling topographic variation in solar radiation in a GIS environment. International Journal of Geographical Information Science, v. 11, n. 5, p. 475-497, 1997. https://doi.org/10.1080/1365881972 42266

Li, H.; Cao, F.; Wang, X.; Ma, W. A. A temperature-based model for estimating monthly average daily global solar radiation in China. The Scientific World Journal, v. 2014, Article ID 128754, 2014. https://doi.org/10.1155/2014/128754

Lima, F. J. L. Avaliação do potencial eólico no Estado da Paraíba: um estudo observacional e numérico. Campina Grande: Universidade Federal de Campina Grande, 2011. (Dissertação de mestrado).

Lima, R. Q. Identificação de parâmetros pelo Método dos Mínimos Quadrados Não Linear. Rio de Janeiro: Pontifícia Universidade Católica, 2009. (Relatório de iniciação científica). Disponível em: <http://www.puc-rio.br/pibic/relatorio_ resumo2009/relatorio/mec/roberta.pdf>. Acesso: 28 out. 2018.

Liu, J.; Liu, J.; Linderholm, H. W.; Chen, D.; Yu, Q.; Wu, D.; Haginoya, S. Observation and calculation of solar radiation on the Tibetan Plateau. Energy Conversion and

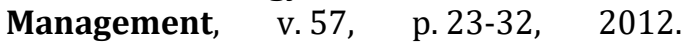
https://doi.org/10.1016/j.enconman.2011.1 2.007

Luo, W.; Taylor, M.; Parker, S. A comparison of spatial interpolation methods toestimate continuous wind speed surfaces using irregularly distributed data from englandand wales. International Journal of Climatology, v. 28, n. 7, p. 947-959, 2008. https://doi.org/10.1002/joc.1583

Menezes, H. E. A. Influência da Zona de Convergência Secundária do Atlântico Sul sobre a ocorrência de precipitação no leste do Nordeste brasileiro. Campina Grande: Universidade Federal de Campina Grande, 2010. (Tese de doutorado).
Meza, F.; Varas, E. Estimation of mean monthly solar global radiation as a function of temperature. Agricultural and Forest Meteorology, v. 100, n. 2/3, p. 231-241, 2000. https://doi.org/10.1016/S0168-1923 (99)00090-8

Nascimento, R. L. Energia solar no Brasil: situação e perspectivas. Brasília: Câmara Federal, 2017. (Estudo técnico.). Disponível em: <http://bd.camara.gov.br/bd/bitstream/ handle/bdcamara/32259/energia_solar_limp .pdf>. Acesso em: 31 out. 2018.

Okundamiya, M. S.; Emagbetere, J. O.; Ogujor, E. A. Evaluation of various global solar radiation models for Nigeria. International Journal of Green Energy, v. 13, n. 5, p. 505512, 2016. https://doi.org/10.1080/15435 075.2014 .968921

Oliveira, S. S.; Souza, E. P. Análise de modelos de mesoescala para caracterização do potencial eólico do Estado da Paraíba. Revista Brasileira de Meteorologia, v. 32, n. 2, p. 277-291, 2017.

QGIS Development Team. QGIS Geographic Information System. Version 2.14. 2014. Open Source Geospatial Foundation Project. Disponível em: <http://qgis.osgeo.org>. Acesso: 28 out. 2018.

Saffaripour, M. H.; Mehrabian, M. A.; Bazargan, H. Predicting solar radiation fluxes for solar energy system applications. International Journal of Environmental Science and Technology, v. 10, n. 4, p. 761768, 2013. https://doi.org/10.1007/s13762013-0179-2

Santos, C. M.; Souza, J. L.; Ferreira-Junior, R. A.; Tiba, C.; Melo, R. O.; Lyra, G. B.; Teodoro, I.; Lyra, G. B.; Lemes, M. A. M. Modelling global solar irradiation using air temperature for Alagoas State, Northeastern Brazil. Energy, v. 71, p. 388-398, 2014. https://doi.org/ 10.1016/j.energy.2014.04.116

Shepard, D. A two-dimensional interpolation function for irregularly-spaced data. Proceedings of the 1968 23rd ACM National Conference, p. 517-524, 1968.

Silva, A. S. A. Ferramentas para modelagem e interpolação de dados ambientais em escala regional. Recife: Universidade Federal Rural de Pernambuco, 2015. (Tese de doutorado). Disponível em: <http://www.ppgbea.ufrpe.br/sites/www.p pgbea.ufrpe.br/files/documentos/tese_anton io_samuel_alves_da_silva.pdf $>$. Acesso em: 30 out. 2018. 
Silva, C. R.; Silva, V. J.; Alves Júnior, J.; Carvalho, H. P. Radiação solar estimada com base na temperatura do ar para três regiões de Minas Gerais. Revista Brasileira de Engenharia Agrícola e Ambiental, v. 16, n. 3, p. 281-288, 2012. https://doi.org/ 10.1590/S1415-43662012000300008

Silva, M. A. V. Meteorologia e Climatologia. 2. ed. Brasília: INMET, 2001.

Souza, A P.; Escobedo, F.J.; Dal Pai, A.; Gomes, E. N. Estimativas das componentes da radiação solar incidente em superfícies inclinadas baseadas na radiação global horizontal. Revista Brasileira de Engenharia Agrícola e Ambiental, v. 15, p. 277-288, 2011. https://doi.org/10.1590/ S1415-43662011000300009

Souza, A.; Santos, D. A. S.; Aristone, F.; KovačAndrić, E.; Matasović, B.; Pires, J. C.; Ikefuti, P. V. Impacto de fatores meteorológicos sobre as concentrações de ozônio modelados por análise de séries temporais e métodos estatísticos multivariados. Holos, v. 5, p. 1-16, $2017 . \quad$ https://doi.org/ 10.15628/holos.2017.5033
Souza, A. P.; Zamadei, T.; Monteiro, E. B.; Casavecchia, B. H. Transmissividade atmosférica da radiação global na Região Amazônica de Mato Grosso. Revista Brasileira de Meteorologia, v. 31, n. 4, p. 639-648, 2016. https://doi.org/10.1590/ 0102-7786312314b20150147

Umoh, M. D.; Udo, S. O.; Udoakah, Y. N. Estimating global solar radiation on horizontal surface from the sunshine hours over Port Harcourt, Nigeria. Journal of Electrical and Electronics Engineering Research, v. 6, n. 1, p. 1-5, 2014.

Vianello, R. L.; Alves, A. R. Meteorologia básica e aplicações. 3. ed. Viçosa: UFV, 2004.

Yakubu, D.; Medugu, D. W. Relationship between the global solar radiation and the sunshine duration in Abuja, Nigeria. Ozean Journal of Applied Sciences, v. 5, n. 3, p. 221-228, 2012. 